Notes on Number Theory and Discrete Mathematics

Print ISSN 1310-5132, Online ISSN 2367-8275

Vol. 27, 2021, No. 4, 276-279

DOI: 10.7546/nntdm.2021.27.4.276-279

\title{
Corrigendum to "The Oresme sequence: The generalization of its matrix form and its hybridization process" [Notes on Number Theory and Discrete Mathematics, Vol. 27, 2021, No. 1, 101-111]
}

\author{
Milena Carolina dos Santos Mangueira ${ }^{1}$, \\ Renata Passos Machado Vieira ${ }^{2}$, Francisco Regis Vieira Alves ${ }^{3}$ \\ and Paula Maria Machado Cruz Catarino ${ }^{4}$ \\ ${ }^{1}$ Department of Mathematics, Federal Institute of Education, Science and Techonology \\ of State of Ceara - IFCE, Treze of Maio, Brazil \\ e-mail: milenacarolina24@gmail.com \\ 2 Department of Mathematics, Federal Institute of Education, Science and Techonology \\ of State of Ceara - IFCE, Treze of Maio, Brazil \\ e-mail: re.passosm@gmail.com \\ ${ }^{3}$ Department of Mathematics, Federal Institute of Education, Science and Techonology \\ of State of Ceara - IFCE, Treze of Maio, Brazil \\ e-mail: fregisdifce.edu.br \\ ${ }^{4}$ University of Trás-os-Montes and Alto Douro - UTAD \\ Vila Real, Portugal \\ e-mail: pcatarino23@gmail.com
}

The present Corrigendum contains a list of corrections applicable to the authors' paper [1].

1. In the Introducion part, where was written " $O_{n}=-n 2^{n}$, with $n$ being a positive integer" replace for " $O_{n}=n \cdot 2^{-n}$, for an integer $n \geq 0$ ".

2. For Theorems 2.1, 2.2 and 2.4, where was written:

$$
\text { For } O=\left[\begin{array}{cc}
1 & -\frac{1}{4} \\
1 & 0
\end{array}\right] \text { we have that: } O^{n}=\left[\begin{array}{cc}
2 O_{n+1} & -\frac{1}{2} O_{n} \\
2 O_{n} & -\frac{1}{2} O_{n-1}
\end{array}\right]^{n}, n \geqslant 1
$$




$$
\begin{aligned}
& \text { For } O=\left[\begin{array}{cc}
0 & 1 \\
-\frac{1}{4} & 1
\end{array}\right] \text {, we have that: } O^{n}=\left[\begin{array}{cc}
-\frac{1}{2} O_{n-1} & 2 O_{n} \\
-\frac{1}{2} O_{n} & 2 O_{n+1}
\end{array}\right]^{n}, n \geqslant 1 . \\
& \text { For } \sigma=\left[\begin{array}{cc}
0 & 1 \\
-4 & 4
\end{array}\right] \text {, we have to: } \sigma^{n}=\left[\begin{array}{cc}
2 O_{-n+1} & -\frac{1}{2} O_{-n} \\
2 O_{-n} & -\frac{1}{2} O_{-n-1}
\end{array}\right]^{n} \text {, for } n>0 .
\end{aligned}
$$

replace with:

$$
\begin{aligned}
& \text { For } O=\left[\begin{array}{cc}
1 & -\frac{1}{4} \\
1 & 0
\end{array}\right] \text {, we have that: } O^{n}=\left[\begin{array}{cc}
2 O_{n+1} & -\frac{1}{2} O_{n} \\
2 O_{n} & -\frac{1}{2} O_{n-1}
\end{array}\right], n \geqslant 1 \\
& \text { For } O=\left[\begin{array}{cc}
0 & 1 \\
-\frac{1}{4} & 1
\end{array}\right] \text {, we have that: } O^{n}=\left[\begin{array}{cc}
-\frac{1}{2} O_{n-1} & 2 O_{n} \\
-\frac{1}{2} O_{n} & 2 O_{n+1}
\end{array}\right], n \geqslant 1 \\
& \text { For } \sigma=\left[\begin{array}{cc}
0 & 1 \\
-4 & 4
\end{array}\right], \text { we have to: } \sigma^{n}=\left[\begin{array}{cc}
2 O_{-n+1} & -\frac{1}{2} O_{-n} \\
2 O_{-n} & -\frac{1}{2} O_{-n-1}
\end{array}\right] \text {, for } n>0 .
\end{aligned}
$$

3. In Definition 2.3, where was written " $n<0$ " replace for " $n>0$ ".

4. In the Properties inherent to matrices section, it is verified that the presented properties are valid for $n=1$ but it is not possible to obtain the same result when modifying $n$. Thus, in this errata, we present properties that are valid for every $n$ assigned:

Property 2.6. For any integer $n, r, 1 \leqslant n<r$, we have:

$$
O_{n+r}=2 O_{n} O_{r+1}-\frac{1}{2} O_{n-1} O_{r} .
$$

Proof. According to Theorem 2.1 and some properties for square matrix exponents, we have:

$$
\begin{aligned}
O^{n+r} & =O^{n} O^{r} \\
{\left[\begin{array}{cc}
2 O_{n+r+1} & -\frac{1}{2} O_{n+r} \\
2 O_{n+r} & -\frac{1}{2} O_{n+r-1}
\end{array}\right] } & =\left[\begin{array}{cc}
2 O_{n+1} & -\frac{1}{2} O_{n} \\
2 O_{n} & -\frac{1}{2} O_{n-1}
\end{array}\right]\left[\begin{array}{cc}
2 O_{r+1} & -\frac{1}{2} O_{r} \\
2 O_{r} & -\frac{1}{2} O_{r-1}
\end{array}\right] .
\end{aligned}
$$

Considering the element $a_{21}$ we have:

$$
\begin{aligned}
2 O_{n+r} & =2 O_{n} 2 O_{r+1}-\frac{1}{2} O_{n-1} 2 O_{r}, \\
O_{n+r} & =2 O_{n} O_{r+1}-\frac{1}{2} O_{n-1} O_{r} .
\end{aligned}
$$

Note 2.7. On Property 2.6, if $n=1$, we have:

$$
\begin{aligned}
O_{n+r} & =2 O_{n} O_{r+1}-\frac{1}{2} O_{n-1} O_{r}, \\
O_{r+1} & =2 O_{1} O_{r+1}-\frac{1}{2} O_{0} O_{r}, \\
O_{r+1} & =2 \frac{1}{2} O_{r+1}-\frac{1}{2} 0 O_{r}, \\
O_{r+1} & =O_{r+1} .
\end{aligned}
$$


Property 2.8. For any integer $m, r, 0<m<r$, we have to:

$$
O_{n+r+1}=2 O_{n+1} O_{r+1}-\frac{1}{2} O_{n} O_{r} .
$$

Proof. According to Property 2.6 and the element $a_{11}$, we have:

$$
\begin{aligned}
2 O_{n+r+1} & =2 O_{n+1} 2 O_{r+1}-\frac{1}{2} O_{n} 2 O_{r}, \\
O_{n+r+1} & =2 O_{n+1} O_{r+1}-\frac{1}{2} O_{n} O_{r} .
\end{aligned}
$$

Note 2.9. On Property 2.8 , if $n=1$, we have to:

$$
\begin{aligned}
O_{n+r+1} & =2 O_{n+1} O_{r+1}-\frac{1}{2} O_{n} O_{r}, \\
O_{r+2} & =2 O_{2} O_{r+1}-\frac{1}{2} O_{2} O_{r}, \\
O_{r+2} & =2 \frac{2}{4} O_{r+1}-\frac{1}{2} \cdot \frac{2}{4} O_{r}, \\
O_{r+2} & =O_{r+1}-\frac{1}{4} O_{r} .
\end{aligned}
$$

Property 2.10. For any integer $n, r, 0<n<r$, we have:

$$
O_{-n-r}=2 O_{-n} O_{-r+1}-\frac{1}{2} O_{-n-1} O_{-r} .
$$

Proof. According to Theorem 2.4 and some properties for square matrix exponents, we have:

$$
\begin{aligned}
\sigma^{n+r} & =\sigma^{n} \sigma^{r} \\
{\left[\begin{array}{cc}
2 O_{-n-r+1} & -\frac{1}{2} O_{-n-r} \\
2 O_{-n-r} & -\frac{1}{2} O_{-n-r-1}
\end{array}\right] } & =\left[\begin{array}{cc}
2 O_{-n+1} & -\frac{1}{2} O_{-n} \\
2 O_{-n} & -\frac{1}{2} O_{-n-1}
\end{array}\right]\left[\begin{array}{cc}
2 O_{-r+1} & -\frac{1}{2} O_{-r} \\
2 O_{-r} & -\frac{1}{2} O_{-r-1}
\end{array}\right] .
\end{aligned}
$$

Considering the left and right elements, we have:

$$
\begin{aligned}
2 O_{-n-r} & =2 O_{-n} 2 O_{-r+1}-\frac{1}{2} O_{-n-1} 2 O_{-r}, \\
O_{-n-r} & =2 O_{-n} O_{-r+1}-\frac{1}{2} O_{-n-1} O_{-r} .
\end{aligned}
$$

Note 2.11. In Property 2.11, if $n=1$, we have:

$$
\begin{aligned}
& O_{-n-r}=2 O_{-n} O_{-r+1}-\frac{1}{2} O_{-n-1} O_{-r}, \\
& O_{-1-r}=2 O_{-1} O_{-r+1}-\frac{1}{2} O_{-2} O_{-r}, \\
& O_{-r-1}=-4 O_{-r+1}+4 O_{-r}, \\
& O_{-r-1}=4 O_{-r}-4 O_{-r+1} .
\end{aligned}
$$

Property 2.12. For any integer $n, r, 0<n<r$, we have:

$$
O_{-n-r+1}=2 O_{-n+1} O_{-r+1}-\frac{1}{2} O_{-n} O_{-r} .
$$


Proof. According to Property 2.10 and and the element $a_{11}$, we have:

$$
\begin{aligned}
2 O_{-n-r+1} & =2 O_{-n+1} 2 O_{-r+1}-\frac{1}{2} O_{-n} 2 O_{-r}, \\
O_{-n-r+1} & =2 O_{-n+1} O_{-r+1}-\frac{1}{2} O_{-n} O_{-r} .
\end{aligned}
$$

Note 2.13. In Property 2.12, if $n=1$, we have:

$$
\begin{aligned}
O_{-n-r+1} & =2 O_{-n+1} O_{-r+1}-\frac{1}{2} O_{-n} O_{-r}, \\
O_{-r} & =2 O_{0} O_{-r+1}-\frac{1}{2} O_{-1} O_{-r}, \\
O_{-r} & =2(0) O_{-r+1}-\frac{1}{2}(-2) O_{-r}, \\
O_{-r} & =O_{-r} .
\end{aligned}
$$

5. And yet, it was possible to identify some references that are not mentioned throughout the text, they are [2-7].

\section{Acknowledgements}

The authors thank to Serpil Halici from Turkey for remarking this correction on October 31, 2021.

\section{References}

[1] Mangueira, M. C. dos S., Vieira, R. P. M., Alves, F. R. V., \& Catarino, P. M. M. (2021). The Oresme sequence: The generalization of its matrix form and its hybridization process. Notes on Number Theory and Discrete Mathematics, 27(1), 101-111.

[2] Bilgici, G., Tokeser, U., \& Ünal, Z. (2017). k-Fibonacci and $k$-Lucas Generalized Quaternions. Konuralp Journal of Mathematics, 5(2), 102-113.

[3] Dasdemir, A., \& Bilgici, G. (2019). Gaussian Mersenne numbers and generalized Mersenne quaternions. Notes on Number Theory and Discrete Mathematics, 25(3), 87-96.

[4] Dasdemir, A. (2016). Generalizations of Modified Pell and Pell Lucas Sequences and Their Generating Matrices and Some Sums. Erzincan University Journal of Science and Technology, 9(3), 178-184.

[5] Koshy, T. (2001). Fibonacci and Lucas Numbers with Applications. [S.l.]. New York: Wiley and Sons Publications, 1.

[6] Sentürk, T. D., Dasdemir, A., Bilgici, G., \& Ünal, Z. (2019). On unrestricted Horadam generalized quaternions. Utilitas Mathematica, 110, 89-98.

[7] Vieira, R., Alves, F. R., \& Catarino, P. (2020). ( $s 1, s 2)$-Padovan matrix sequence and the case of generalization. Analele ştiinţifice ale Universitătii “Al. I. Cuza” din Iaşi Mat. (N.S.) (to appear). 